\title{
Losing weight: easier than before, better than before
}

\section{Opinion}

Nowadays, obesity has become a major global health challenge. Pakistan is the 9 th most obese country of the world. ${ }^{1}$ Obesity can lead to number of chronic diseases such as type II diabetes mellitus (T2DM), atherosclerosis and a spectrum of cardiovascular diseases (CVD) etc. ${ }^{2}$ Therefore, losing weight is of much importance to prevent from these diseases. Dropping a few pounds makes you look and feel better and can increase your overall well-being. ${ }^{3}$ Losing weight is a complex process and it doesn't require solely crash dieting, as crash dieting may help you reach your weight loss goals, but the results are temporary and you will regain the lost weight in a couple of days after you stop dieting. Reason being that in the course of crash dieting, for quicker weight loss results, major food groups is excluded. As a result, nutritional deficiencies are bound to set in. This lowers your immunity and makes you susceptible to various illnesses. Prolonged sustenance on crash dieting will make you lose muscle mass. ${ }^{4}$

There are many ways to lose a lot of weight fast. However, most of them will make you hungry and unsatisfied. But to lose weight effectively and without feeling lethargic, you just have to make few changes in your diet and there you are. Firstly you have to cut back on sugars and starches (carbohydrates) because these are the foods that stimulate secretion of insulin which is the main fat storage hormone in the body. When insulin goes down, fat has an easier time getting out of the fat stores and the body starts burning fats instead of carbohydrates resulting in weight loss. ${ }^{5}$ Secondly, drinking water a half hour before meals increases weight loss due to an indirect effect of less food intake. Studies showed that water consumption of about $500 \mathrm{ml}$ prior to meal results in reduction of meal intake, thereby helping to lose weight. ${ }^{6}$ Another factor that can contribute to weight loss can be making your meals on whole foods. They are healthier, more filling and much less likely to cause overeating. In addition, poor sleep is one of the strongest risk factors for weight gain, so taking care of your sleep is important. It is seen that short sleep duration may be associated with the development of obesity from childhood to adulthood. ${ }^{7}$

Excess body fatness is a result of an imbalance between energy intake and energy expenditure, and physical activity is an extremely important part of a weight management program. Combining exercise with a healthy diet is a more effective way to lose weight than depending on calorie restriction alone. In addition, if you exercise, you lower your risk of developing certain types of cancers such as colon and breast cancer. Exercise is also known to help contribute to a sense of confidence and well-being, thus possibly lowering rates of anxiety and depression. Exercise is helpful for weight loss and maintaining weight loss. Exercise can increase metabolism, or how many calories you burn in a day. It can also help you maintain and increase lean body mass, which also helps increase number of calories you burn each day by keeping the metabolism in check and increasing muscle mass instead of fat deposits. ${ }^{8}$
Volume 5 Issue 4 - 2016

\author{
Zainab Hussain \\ RLAK College of Home Economics, Pakistan
}

Correspondence: Zainab Hussain, RLAK College of Home Economics, Karachi, Pakistan,

Email zainabhussain874@gmail.com

Received: December 16, 2016 | Published: December 29, 2016

Weight management involves adopting a healthy lifestyle that includes knowledge of nutrition and exercise, a positive attitude and the right kind of motivation. Internal motives such as better health, increased energy, self-esteem and personal control increase your chances of lifelong weight management success, overall increasing your immunity, aesthetics, general health and longevity. ${ }^{9}$

\section{Acknowledgements}

I am thankful to my friend Saadullah Yousuf who took out his precious time to edit this manuscript and bring in more clarity.

\section{Conflict of interest}

The author declares no conflict of interest.

\section{References}

1. Pakistan $9^{\text {th }}$ most obese country: Study. The Express Tribune. 2014.

2. Hidekuni Inadera. The usefulness of circulating adipokine levels for the assessment of obesity-related health problems. Int J Med Sci. 2008;5(5):248-262.

3. Surprising Health Reasons to Lose Weight-Weight Loss. Everyday Health.

4. Advantages and Disadvantages of Crash Diets. Crash Diets Homepage.

5. How to lose weight Fast: 3 Simple Steps, Based on Science. Authority Nutrition.

6. Dennis EA, Dengo AL, Comber DL, et al. Water Consumption Increases Weight Loss during a Hypocaloric Diet Intervention in Middle-aged and Older Adults. Obesity. 2009;18(2):300-307.

7. Cappuccio FP, Taggart FM, Kandala NB, et al. Meta Analysis of Short Sleep Duration and Obesity in Children and Adults. Sleep. 2009;31(5):619-626.

8. Hill JO. The Role of Exercise in Weight Maintenance. Obesity Treatment. 1995:127-132.

9. Behavior Modification Ideas for Weight Management. UCSF Medical Center. 